Archives de sciences sociales des religions

151 | juillet-septembre 2010

Fondations des lieux de culte

\title{
Construction de mosquées en Albanie, 1920-1939
}

Nathalie Clayer

\section{OpenEdition}

Journals

Édition électronique

URL : http://journals.openedition.org/assr/22350

DOI : 10.4000/assr.22350

ISSN : $1777-5825$

Éditeur

Éditions de l'EHESS

Édition imprimée

Date de publication : 1 septembre 2010

Pagination : 91-105

ISBN : 978-2-7132-2255-9

ISSN : 0335-5985

Référence électronique

Nathalie Clayer, «Construction de mosquées en Albanie, 1920-1939 », Archives de sciences sociales des religions [En ligne], 151 | juillet-septembre 2010, mis en ligne le 20 octobre 2010, consulté le 19 avril 2019. URL : http://journals.openedition.org/assr/22350 ; DOI : 10.4000/assr.22350 


\section{Nathalie Clayer}

\section{Construction de mosquées en Albanie, 1920-1939}

Au moins une dizaine de mosquées relativement importantes ont été construites dans l'Albanie de l'entre-deux-guerres, ce petit pays qui comprend alors environ un million d'habitants, dont $70 \%$ de musulmans. À la même époque, dans les autres États balkaniques, peu de mosquées sont édifiées (ou pas du tout) - pour des communautés musulmanes minoritaires il est vrai, mais dont certaines sont tout aussi importantes numériquement, comme en Bosnie-Herzégovine. La seule nouvelle mosquée d'importance construite dans la première moitié du XXe siècle est celle de Zagreb, édifiée plus tardivement, à l'époque du régime croate oustachi, pendant la Seconde guerre mondiale (Hasanbegović, 2007). En Turquie, autre pays à majorité musulmane issu de l'Empire ottoman dans la région, les années vingt-trente ne sont pas non plus favorables à la construction de nouveaux lieux de culte musulman. Au contraire : sous la houlette d'Atatürk, des restrictions sont imposées à l'édification de mosquées et certaines d'entre elles seront réaménagées pour une utilisation profane (Yavuz, 2003). L'exemple le plus significatif est la transformation en musée, au milieu des années trente, d'Aya Sofya, l'église Sainte-Sophie, devenue à l'époque ottomane l'un des lieux de culte musulman les plus prestigieux d'Istanbul (Necipoğlu, 1982).

Pourquoi des constructions de mosquées en Albanie à cette époque, alors que le pays est déclaré laïque, à l'image de la Turquie, et qu'il traverse une crise économique aiguë ? Quels sont les enjeux et les acteurs de ces entreprises ? Avant d'apporter des éléments de réponse à travers l'analyse de quelques cas spécifiques d'édification de mosquées, à Durrës, Saranda et Tirana, précisons quelques éléments du contexte religieux et politique qui caractérisent alors le jeune État albanais.

\section{Un État majoritairement musulman à la souveraineté fragile}

L'Albanie est un État issu du démembrement de l'Empire ottoman en Europe, consécutif aux guerres balkaniques de 1912-1913. Sa souveraineté est rétablie après la Première Guerre mondiale, synonyme d'occupations grecque, française, italienne et austro-hongroise. L'entre-deux-guerres est une période de passage de l'Empire à l'État-nation, période de construction étatique et d'affirmation 
d'une souveraineté qui demeure fragile en raison des aspirations territoriales grecques, yougoslaves et surtout italiennes (Fischer, 1984). Durant cette période, l'Albanie devient, en effet, au moins économiquement, une semi-colonie italienne (Sjöberg, 1991 ; Morrozo della Rocca, 1990), avant d'être annexée, en 1939, par Mussolini.

Le rapport entre islam et construction étatique y est fortement marqué par une situation de contact à la fois interne et externe. Les musulmans du pays voisinent avec des non-musulmans - plus ou moins selon les régions : essentiellement des chrétiens orthodoxes dans le Centre et le Sud du pays (représentant environ $20 \%$ de la population totale) et des catholiques au Nord (environ $10 \%$ de la population). En outre, le pays et sa majorité musulmane sont en contact externe avec des États tous majoritairement chrétiens (Grèce, Royaume de Yougoslavie et Italie). La Turquie, elle aussi majoritairement musulmane, n'a pas de frontière commune avec l'Albanie.

Or l'identité musulmane de l'Albanie ne va pas de soi, même si les musulmans y sont majoritaires. C'est en tout cas le sentiment des hommes politiques et des intellectuels albanais, musulmans et non-musulmans. Certains d'entre eux n'hésitent pas à déclarer que les Albanais musulmans doivent revenir à la religion (chrétienne) de leurs ancêtres, l'islam étant associé à l'ancienne domination ottomane. Une souveraineté musulmane en Europe pourrait poser des problèmes de légitimité du point de vue des pays voisins. Elle incommode également des intellectuels et des administrateurs musulmans locaux ayant intériorisé l'idée européo-centrée d'un islam obstacle au progrès et à la civilisation.

Au reste, le rapport entre islam et construction nationale albanaise s'est avéré problématique dès avant la création d'un État albanais. Avant 1912, légitimer une nation majoritairement musulmane en Europe avait conduit à des définitions areligieuses de l'identité albanaise. "La religion des Albanais est l'albanité ", "un Albanais est albanais avant d'être musulman, orthodoxe ou catholique " étaient des slogans fréquemment utilisés par les albanistes à l'intérieur comme à l'extérieur de l'Empire ottoman, plus particulièrement à l'adresse des Européens. Pour les mêmes raisons, le bektachisme, une forme mystique de l'islam, était également mis en avant, afin de présenter les musulmans albanais comme des " musulmans autres ", des musulmans non fanatiques, proches des chrétiens, contrairement aux musulmans turcs (Clayer, op. cit.)

À l'issue de la Première Guerre mondiale, dans l'État albanais nouvellement reconnu par la communauté internationale, la politique des dirigeants est en conséquence assez proche de celle de la Turquie. L'État y est proclamé sans religion officielle, laïque (afetar en albanais). Mais à y regarder de près, comme en Turquie, cette laïcité n'est pas celle de la France de l'époque. Elle est plutôt assez proche du système du Concordat français au XIX ${ }^{\mathrm{e}}$ siècle, caractérisé par la laïcité de l'État, l'égalité des cultes reconnus, une police des cultes et une politique religieuse, si l'on reprend l'analyse qu'en fait Jean-Marie Mayeur dans La question 
laïque (1997). Ce qui signifie une volonté de mise sous tutelle des «Églises» reconnues par l'État, une volonté d'intervention de l'État dans l'«administration " des cultes, une ingérence des Églises dans la vie de la nation, ainsi qu'une reconnaissance de l'utilité sociale de la religion (ibid.)

Dans ce cadre, l'institutionnalisation et la nationalisation des communautés religieuses musulmanes sont mises en œuvre autour d'une hiérarchie de muftiscadis déjà établie par les autorités d'occupation austro-hongroises lors du premier conflit mondial (San Nicolo, 1918 : 253-268). Avec l'introduction du Code civil en 1928-1929, qui entraîne l'abolition des tribunaux de la charia et la suppression de leurs prérogatives en matière judiciaire, les muftis deviennent davantage des administrateurs des affaires religieuses, puisque les fetvalfatwa ne peuvent plus être délivrées que par une institution centrale. Plus généralement, les institutions religieuses islamiques sont placées sous un certain contrôle des autorités politiques, qui interviennent fréquemment dans les orientations de la Communauté, la nomination ou la destitution des cadres religieux, etc. Une subvention annuelle de l'État est fixée pour compenser l'insuffisance des revenus des waqf-s et dons des fidèles. Par ailleurs, le gouvernement albanais entreprend la séparation des Églises et de l'École, avant un revirement, en 1937, lorsque le roi décide de réintroduire l'enseignement de la religion à l'École, afin de lutter contre le "danger communiste ». Il affaiblit aussi, dans une certaine mesure, les institutions islamiques en reconnaissant officiellement, au début des années trente, l'autonomie de la confrérie mystique des Bektachis.

Toutefois, la résistance à la centralisation et aux réformes imposées d'en haut est importante, et l'autonomie des acteurs locaux ou leur propre façonnement des institutions loin d'être négligeable. Un exemple caractéristique est celui des membres de la confrérie mystique des Tidjanis qui s'opposent à certaines réformes de l'enseignement religieux, ainsi qu'à la désignation de candidats du gouvernement pour le congrès des musulmans du pays, organisé en 1929. En 1930, ils sont intégrés dans les institutions islamiques centrales afin d'être neutralisés. Mais leur intégration contribue à une certaine revivification de l'islam albanais dans les années suivantes (Clayer, 2009b). En outre, les autorités politiques ne manquent pas, durant cette période, d'utiliser l'islam, et la religion en général, dans un souci de contrôle social, en particulier contre l'éventuelle montée d'une opposition communiste (surtout à partir des années trente). Il en résulte une politique religieuse plus complexe que le paradigme de la laïcisation ne le laisse entendre, comme nous le verrons dans le processus d'édification de mosquées.

\section{Construire ou fermer des mosquées ?}

Une recherche non systématique menée dans le fonds de la Communauté islamique et dans les fonds du ministère de l'Intérieur montre que, dans les années vingt, de multiples demandes émanent de la population musulmane pour la libération de mosquées occupées par l'armée, bien après le premier conflit mondial. 
D'autres expriment le besoin de lieux de culte lorsque des mosquées ont été détruites pendant les conflits et périodes d'occupation, lors de l'occupation grecque du sud du pays par exemple. Des mosquées devaient remplacer celles tombées en ruine, en particulier du fait de la perte des biens de mainmorte (vakf-s) qui leur étaient attachés, passés en dehors du territoire national à la suite du découpage fait par les Grandes Puissances. Enfin, des fidèles musulmans réclamaient de nouveaux lieux de culte en remplacement des quelques mosquées détruites dans les années vingt par les autorités locales albanaises en raison de l'application de plans d'urbanisme. Du côté des autorités religieuses islamiques, la question des lieux de culte était aussi sensible, puisque, à plusieurs reprises, elles demandent à leurs branches régionales d'établir des états des lieux et des contrôles. Il s'agit de dénombrer les mosquées et de vérifier leur condition. Dans certaines régions du Sud du pays, les muftis locaux relaient les demandes de la population musulmane. Dans un cas, le mufti suggère lui-même d'édifier des mosquées afin de reconquérir des populations liées à la confrérie des Bektachis ${ }^{1}$.

La volonté de certains groupes de musulmans et celle des autorités religieuses semblent donc se rejoindre pour re-densifier le réseau des mosquées dans le pays - sans que de véritables initiatives ne soient d'ailleurs toujours prises pour leur construction en raison de contraintes financières notamment. Néanmoins, vers la fin de la décennie en question, une autre tendance apparaît au sein des instances islamiques centrales. En février 1927, un débat est en effet lancé par le Directeur Général des Vakfs, Salih Vuçitern (ou Vuçiterni), un homme proche des autorités politiques et jouant un rôle important dans les institutions islamiques. Il s'agit d'un débat au sujet des réformes à entreprendre pour améliorer la situation des musulmans albanais, "entourés de peuples de culture ». Salih Vuçitern propose, en particulier, de supprimer les madrasas locales pour concentrer les efforts sur un ou deux établissements d'enseignement religieux dans le pays. Il propose, en outre, de supprimer les mosquées qu'il considère " inutiles ». Il ne précise pas ce qu'il entend par " inutiles ", mais d'après certains documents on comprend que la Communauté islamique est poussée par le gouvernement albanais à fermer des mosquées peu fréquentées ou mal entretenues, par exemple dans des centres urbains où se trouvent déjà d'autres mosquées ${ }^{2}$. De même que

1. Voir Arkivi Qendror i Shtetit (AQSh, Archives centrales de l'État), Tirana, Fonds 882 (Communauté musulmane), année 1923, dossier 74, année 1924, dossier 85 ; année 1926, dossier 14 ; année 1931, dossiers 105 et 117 ; F. 152 (ministère de l'Intérieur), année 1923, dossier 377, folio 2-4; année 1925, dossier 59; année 1930, dossier 459, folio 73. Dans les années trente, des demandes de construction de mosquées émanèrent aussi de certains groupes d'immigrés venant du Kosovo, installés dans des villages en Albanie centrale (AQSh, F. 882, année 1935, dossier 58).

2. Cette pression avait pour but de diminuer le déficit budgétaire de la Communauté islamique (voir AQSh, F. 155, ministère de la Justice, année 1929, dossier VIII-105, folios 17-18 et année 1932, dossier VIII-227, folio 3). On aurait ainsi fait passer le nombre de mosquées de plus de 120 à 58 ! 
pour les madrasas, il s'agit pour Salih Vuçitern et certains de ses collègues d'adapter l'islam à l'Europe, et donc de résister à la pression des chrétiens locaux et étrangers par l'éducation et la rationalisation, à la fois dans l'interprétation des textes et dans l'organisation de la communauté (Vuçitern, 1927).

Dans les années qui suivirent, plusieurs grandes mosquées furent pourtant édifiées sur le sol albanais et présentées comme les principales réalisations de la Direction de la Communauté islamique. Qui en furent exactement les promoteurs et dans quels buts?

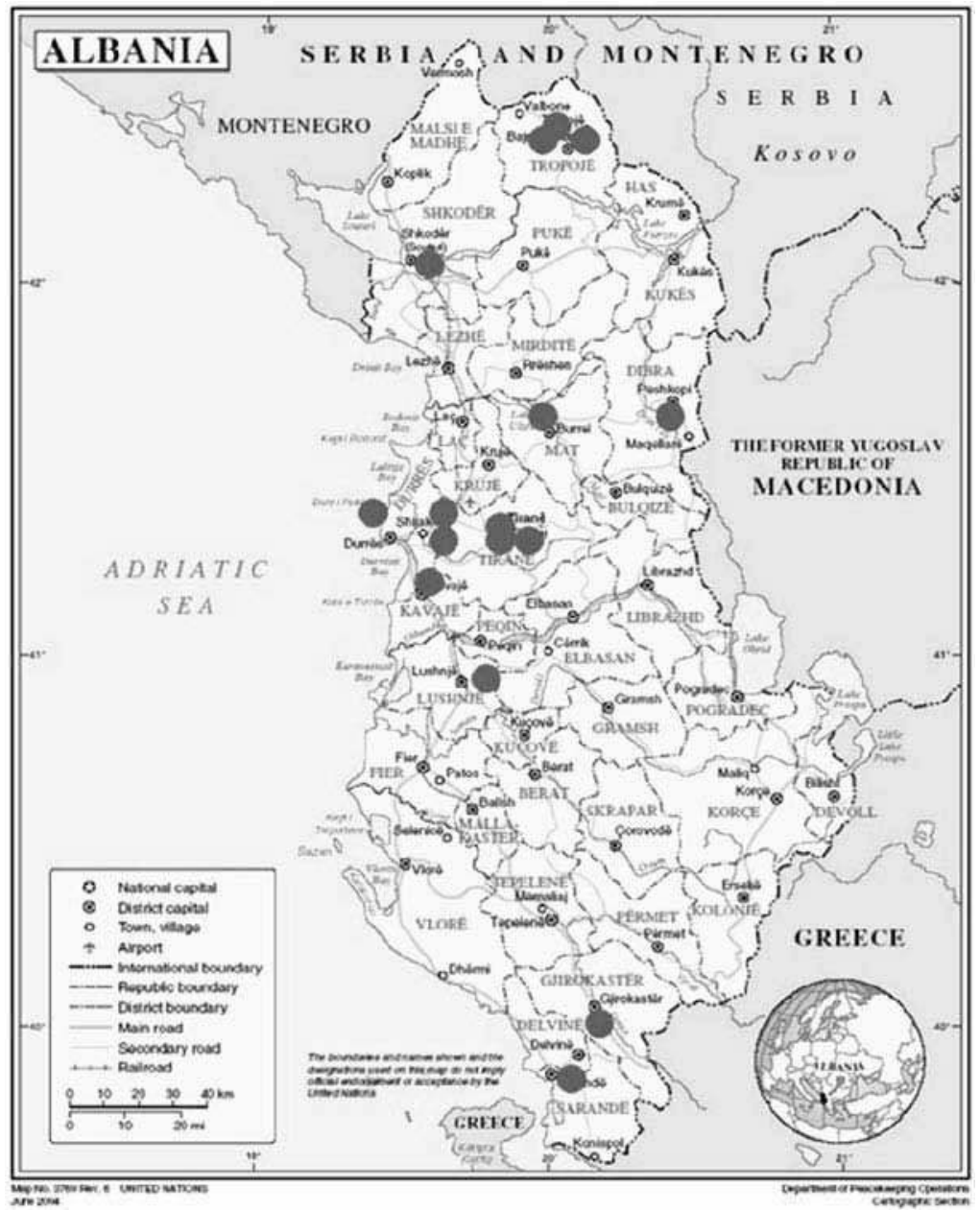

Nouvelles mosquées construites en Albanie dans l'entre-deux-guerres 


\section{Durrës : prestige et réforme de l'islam}

À l'origine de la mosquée de Durrës, le plus grand port du pays situé à une quarantaine de kilomètres de la capitale, se trouve Salih Vuçitern lui-même ${ }^{3}$. Quelques mois après avoir expliqué qu'il fallait supprimer les mosquées « inutiles ", le Directeur Général des Vakfs demande en effet à la Direction de la Communauté islamique qu'une grande mosquée soit édifiée à Durrës, qui puisse rassembler toute la population de la ville; une mosquée avec une kubbe, c'est-à-dire une coupole, et deux minarets, donc un bâtiment prestigieux. Sali Vuçitern donne pour cela deux arguments. Durrës est destiné à devenir le principal port d'Albanie, future porte d'entrée et de sortie vers l'extérieur. On comprend qu'il s'agit par là de donner une certaine image de l'islam et des musulmans albanais à ceux qui débarquent dans le pays en provenance d'Europe. D'autre part, pour Salih Vuçitern, le but est aussi de défendre la dignité des musulmans face aux grandes églises édifiées par les autres communautés dans la ville. L'édifice du culte devait permettre de restaurer le prestige de la communauté musulmane locale face aux chrétiens qui avaient su, au cours des décennies précédentes, se doter de lieux de culte perçus comme "grands » et "modernes ». La mosquée devait justement pouvoir accueillir " tous les musulmans de la ville ». Il s'agissait donc de donner une autre dimension à la communauté locale : l'islam n'était plus un islam de quartier, mais un islam communautaire à l'échelle de la ville et de la région, voire de la nation.

La construction de cette mosquée ne démarre qu'en 1931, à une époque où la communauté islamique vient d'être réorganisée sous les auspices du roi Zog et du gouvernement albanais. Au reste, il est désormais prévu que le nouvel édifice porte le nom du Roi, preuve que l'édifice est bien associé à l'échelle de la nation et à son souverain. D'autre part, le futur lieu de culte est alors étroitement associé à l'idée de réforme de l'islam. La pose de la première pierre est, en particulier, l'occasion pour différents groupes d'exprimer leur désir de réforme de l'islam que l'édifice est censé symboliser. Ainsi, des administrateurs musulmans réformateurs regroupés autour du journal Besa utilisent-ils un article sur l'évènement comme prétexte à l'affirmation de la nécessité des réformes. Selon les rédacteurs du journal, les Albanais ne devaient pas imiter les autres, mais être imités ; il leur fallait s'adapter à la mentalité moderne malgré les difficultés ; le pays étant situé au beau milieu de l'Europe, il devait mener des réformes libérales, sur les plans à la fois législatif, social et mental, afin d'arriver à la civilisation (Besa, 11/8/1931:2). La cérémonie d'inauguration des travaux est également l'occasion pour un représentant de la jeunesse musulmane de demander aux responsables des institutions religieuses islamiques des réformes dogmatiques. C'était toutefois aller trop loin pour ces institutions qui, tout en approuvant

3. Voir AQSh, F. 882, année 1928, dossier 44, folio 14. 
les réformes que la future mosquée symbolisait, refusaient que l'on puisse toucher au dogme (Sharofi, 1931).

Outre l'idée de réforme, la mosquée de Durrës est, dès l'origine, associée à l'idée de modernité. Alors que les travaux de construction sont presque achevés, le chef de la communauté islamique albanaise s'empresse, par exemple, de signaler dans son rapport annuel au gouvernement qu'il a donné l'autorisation de détruire le restaurant qui se trouve au pied des marches et de construire à sa place une petite boutique qualifiée de " moderne " ${ }^{4}$. Il faut souligner que le style de cette mosquée, achevée en 1938, est résolument " moderne », ne serait-ce que par l'emploi du béton, et tranche sur celui des mosquées édifiées à l'époque ottomane. Ces dernières étaient de deux types : les plus anciennes, construites selon le type ottoman classique, avec une base carrée surmontée d'une coupole et un portique à trois dômes ; les plus récentes, bâties sur un plan rectangulaire, avec des plafonds en bois souvent non surmontés d'une coupole, et des murs richement décorés, selon un modèle très courant dans les Balkans. La nouvelle mosquée tranchait à la fois par sa taille, par l'emploi du béton, par le retour à la coupole, par le portique à colonnes, ainsi que par l'ajout d'un étage intermédiaire entre la coupole et le portique.

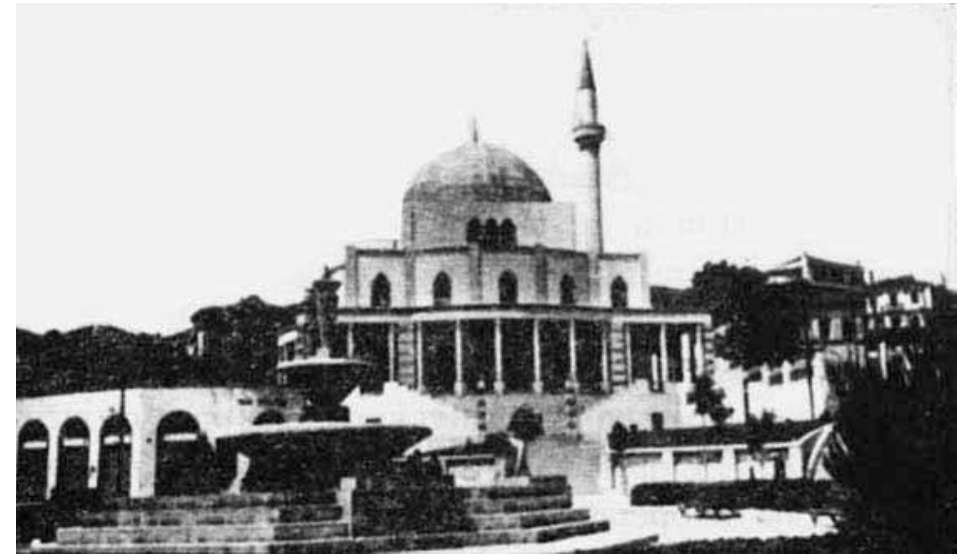

Mosquée de Durrës (Source : Collection privée NC)

Pour les dirigeants de la communauté islamique et le gouvernement, comme pour les administrateurs et la jeunesse musulmane, la construction de cet édifice relève donc du rétablissement du prestige de l'islam, tant vis-à-vis des pays chrétiens voisins que vis-à-vis des chrétiens locaux.

4. AQSh, F. 882, année 1937, dossier 1, rapport de Behxhet Shapati sur l'activité de la CI du $1 / 5 / 1937$. 


\section{Saranda : nation et souveraineté du territoire}

Une mosquée est édifiée dans un autre port, plus au sud, sur la côte adriatique, à Saranda, non loin de la frontière grecque. Cet édifice, plus modeste que le précédent, n'en est pas moins symbolique, même si au départ sa construction répond au simple besoin des fidèles musulmans.

Dès 1922, c'est la population musulmane locale qui demande la construction d'une mosquée ou, plus exactement semble-t-il, la possibilité d'utiliser un baraquement comme mosquée. Comme les baraquements sont propriétés de l'État, cette demande est transmise par le Grand Mufti au Premier ministre. Or, l'argument utilisé par le Grand Mufti pour appuyer la demande relève des rapports entre communautés religieuses. Il explique que la population orthodoxe locale possède une église pour prier, tandis que les musulmans, supérieurs en nombre (selon lui), n'ont aucun lieu de culte dans ce petit port qui ne compte alors que quelques centaines d'habitants. Malgré un rapport défavorable du préfet remplaçant, selon lequel il ne vaut pas la peine que l'État albanais dépense de l'argent ou se dépouille d'un baraquement pour une mosquée qui ne servirait qu'à une poignée de musulmans et qui risquait de provoquer des réactions hostiles de la part de la Grèce, l'affaire est traitée par le Conseil des Ministres, le 30 décembre 1922. Il est décidé de permettre provisoirement l'usage d'une baraque pour faire la prière, en attendant que le problème des baraquements soit réglé 5 .

En fait, l'optique des autorités politiques ne se situe pas au niveau local. Dès cette époque, le gouvernement albanais cherche à " albaniser » ce petit port qui se trouve à la frontière grecque, face à Corfou, pour en éliminer le caractère grec, dû à la présence dans la ville et ses alentours d'une population grecque et/ou grécophone, et contrer le danger d'expansionnisme de l'État grec. Les autorités albanaises mettent alors en place une politique d'installation de réfugiés albanais musulmans venant de Grèce, et facilitent l'installation de commerçants et d'entrepreneurs albanais musulmans, venant en particulier de Gjirokastër ${ }^{6}$. L'ouverture d'un lieu de culte musulman, serait-ce provisoire, devait participer de cette inscription dans la ville d'une présence à la fois " musulmane " et " albanaise ». Toutefois, certains administrateurs locaux, réticents, s’y opposent. En 19271928, un comité local se forme autour d'un célèbre cheikh qui fait partie de ces musulmans réfugiés dans la région, pour la construction d'une vraie mosquée. De son côté, l'État albanais donne un terrain, non sans avoir offert parallèlement un terrain à la communauté catholique, dans une autre région du pays ${ }^{7}$.

5. AQSh, Fonds 152 (ministère de l'Intérieur), année 1924, dossier 398, folio 4, rapport du préfet remplaçant, Gjirokastër, 1/7/1922 ; folio 8, lettre du Grand Mufti au ministre, Tirana, le 22 octobre 1924 (qui indique qu'en réalité, aucun baraquement n'a été cédé aux musulmans pour la prière, contrairement à la décision du Conseil des Ministres).

6. AQSh, F. 152, année 1921, dossier 41.

7. AQSh, F. 882, année 1928, dossier 28. 
La construction de l'édifice ne débute cependant qu'en $1936^{8}$, pour des raisons financières probablement. Or le financement est assuré en partie grâce à un don d'une sœur du roi Zog. Comme à Durrës, la construction de la mosquée est associée à la nouvelle famille régnante. Dans la cadre d'une campagne d'albanisation des toponymes, la ville est d'ailleurs rebaptisée Zogaj, d'après le nom du souverain (Clayer, 2009a). Elle est ainsi doublement associée, par son nom et par la bienfaitrice de la mosquée, à la dynastie. De ce fait, la présence de la mosquée participe de l'albanisation du territoire face aux revendications grecques, associées, elles, à l'orthodoxie.

Cet amalgame était fait du côté adverse, si l'on en juge par un texte publié par le club vorio-épirote d'Athènes et du Pirée, c'est-à-dire du club rassemblant des émigrés chrétiens orthodoxes venant du Nord de l'Épire, autrement dit du sud de l'Albanie. Dans le texte en question, ces chrétiens du Sud albanais réagissaient, entre autres, contre l'albanisation de l'Épire du Nord et demandaient concrètement : « Qui va empêcher les Turco-Albanais de construire une mosquée imposante à Saranda où il n'y a aucun musulman ? » ${ }^{9}$. Pour eux, la mosquée était bien le symbole de l'islamisation, et donc de l'albanisation de l'Épire du Nord.

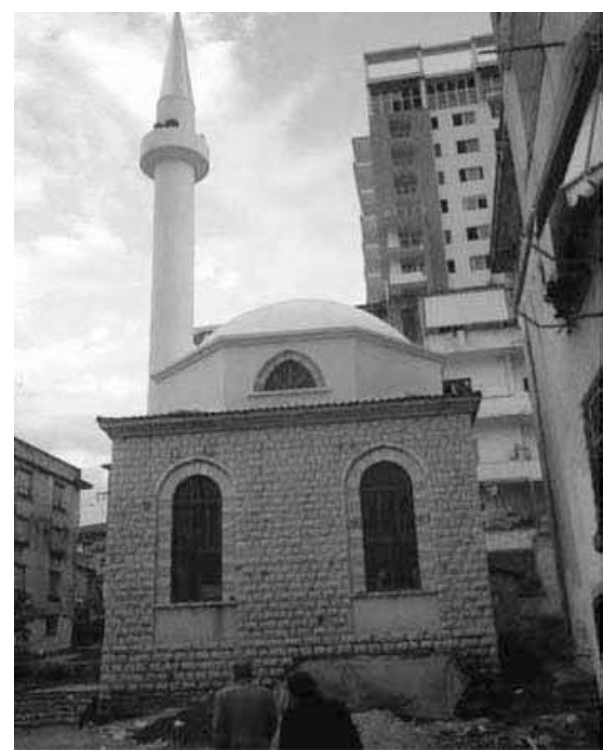

Mosquée de Saranda (Source : http://3.bp.blogspot.com/_2gHIijV_2MQ/ R6jAr3JVBtI/AAAAAAAAAT8/sGOSIUTTm6s/s1600-h/xhamia+sarande.jpg)

8. Voir note 4.

9. AQSh, F. 195 (ministère de l’Éducation), année 1937, dossier 22. 
Dans cette région, les enjeux de la fondation ou de la rénovation d'églises orthodoxes étaient également très liés à la construction étatique albanaise telle qu'elle se faisait face aux revendications grecques-orthodoxes. Pour le gouvernement, ainsi que pour les milieux soutenant la création d'une Église orthodoxe albanaise autocéphale, il s'agissait de symboliser cette nouvelle Église nationale par le biais d'un style architectural considéré comme " albanais " et non " étranger ", ou encore par l'exclusion de l'utilisation d'écrits en caractères grecs et de la couleur bleue ciel, perçue comme couleur nationale grecque ${ }^{10}$.

\section{Tirana : initiatives individuelles et institutions islamiques}

Cette politique d'affirmation d'un islam réformé face à l'Europe et aux chrétiens locaux, ou d'affirmation de la souveraineté du territoire albanais face à la Grèce à travers la construction de lieux de culte musulmans semble avoir été difficilement mise en œuvre. En témoignent les délais qui se sont écoulés entre la décision de construire ces mosquées, vers 1928, et leur achèvement une dizaine d'années plus tard. De fait, le gouvernement albanais et les institutions religieuses islamiques n'ont pas les moyens financiers de mener à bien ces constructions plus rapidement, dans un contexte de crise économique aiguë. Le cas de Tirana, la nouvelle capitale désignée comme telle seulement au début des années vingt, montre comment plusieurs nouvelles mosquées ou restaurations d'anciens édifices de culte musulman sont en réalité réalisées grâce aux fidèles, ce qui n'empêche pas les institutions religieuses d'utiliser ces constructions pour la légitimation d'un islam réformé, ainsi que pour leur propre légitimation.

La population de Tirana croît de façon notable au cours de l'entre-deuxguerres en raison du nouveau rôle politique et administratif de la ville. Dans ce contexte, au début des années trente, grâce aux habitants de plusieurs quartiers, trois ou quatre mosquées y sont construites ou reconstruites. Or, les institutions islamiques, qui n'ont pas les moyens de lancer elles-mêmes ces constructions, semblent s'emparer de ces investissements individuels ou collectifs locaux. La revue de la communauté ne manque pas de publier des articles au sujet de l'inauguration des nouveaux édifices ${ }^{11}$. Ses rédacteurs félicitent les comités initiateurs et saluent leurs sentiments religieux. En particulier, l'une des mosquées, construite dans un "style moderne ", sur un nouveau boulevard de la capitale, est particulièrement mise en avant par les dirigeants religieux de la capitale. Il s'agit de souligner que la modernisation matérielle de la ville ne peut se faire sans une composante religieuse. Une photo de la nouvelle mosquée apparaît aussi dans le journal Besa ( ${ }^{\circ}$ 623, 9/8/1933 : 3) déjà mentionné, alors que ce périodique ne comporte pratiquement aucune illustration photographique. Elle

10. Voir par exemple AQSh, F. 152, année 1930, dossier 187, folio 49, 51, 61.

11. Zani i naltë, VIII/3-4, novembre-décembre 1932, pp.483-85; VIII/12, août 1933, pp. $742-748$. 
est aussi censée symboliser cet islam réformé et moderne dont le journal s'est fait le promoteur au sujet de la future mosquée de Durrës.

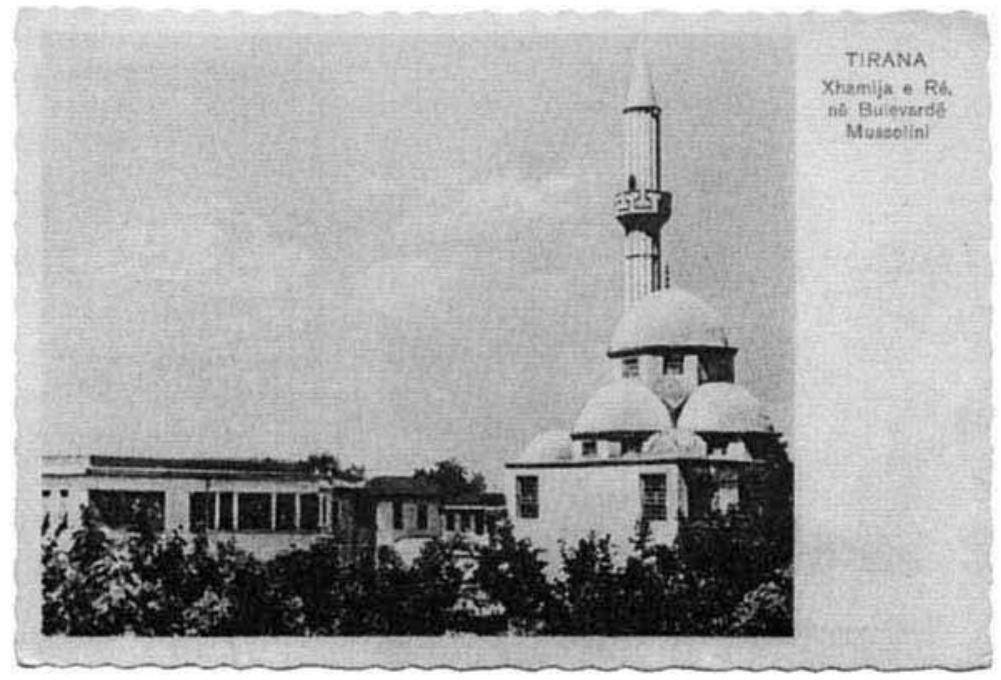

La Nouvelle Mosquée, Boulevard Mussolini, d'après une carte postale (Source: Tirana in postcards till the year' '44, s.l.n.d. : 89)

Dans les colonnes de la revue de la communauté islamique, l'appel aux fidèles revient d'ailleurs régulièrement. Pour cela, des exemples de dons sont signalés, et l'assurance d'être récompensé par Dieu est soulignée ${ }^{12}$. Il en va du prestige de l'islam dans le pays, mais aussi du prestige des institutions religieuses. Les rapports du chef de la communauté islamique adressés au gouvernement mettent systématiquement en avant le progrès des constructions de lieux de culte pour justifier de l'action de la communauté ${ }^{13}$.

En juin 1938, un article anonyme est encore publié dans la revue afin de pousser la population à faire œuvre de bienfaisance pour financer la construction d'une grande mosquée moderne centrale, pour les grandes cérémonies dans la capitale. La vieille mosquée de Sulejman Pasha est considérée comme trop petite et inadaptée aux besoins de l'époque. Il n'y a pas suffisamment de place pour les cérémonies " religieuses et nationales ». Or l'auteur de l'article considère que cela fait "mauvais effet » sur les gens ${ }^{14}$. C'est-à-dire qu'il en va du prestige de l'islam dans l'opinion publique. Pourtant, une telle mosquée ne vit pas le jour à

12. Zani $i$ naltë, IX/1, septembre 1933 , p. 32 ; IX/2-3, octobre-novembre 1933, pp. 76 77 ; X/5, mai 1935, pp. 159-160.

13. AQSh, F. 882, année 1932, dossier 2, rapport de Behxhet Shapati, du 1/5/1933 ; année 1938, dossier $n^{\circ} 4$, rapport du 1/5/1938.

14. "Kyeqyteti ka nevojë për nji Xhai te madhe e moderne " [La capitale a besoin d'une mosquée grande et moderne], Zani $i$ naltë, XIII/6, juin 1938, pp. 178-179. 
Tirana, contrairement à Shkodra où les autorités religieuses locales et la population musulmane firent un effort suffisant pour édifier une grande mosquée, achevée sous l'occupation italienne (Bushati, 1998 : 125-127) ${ }^{15}$.

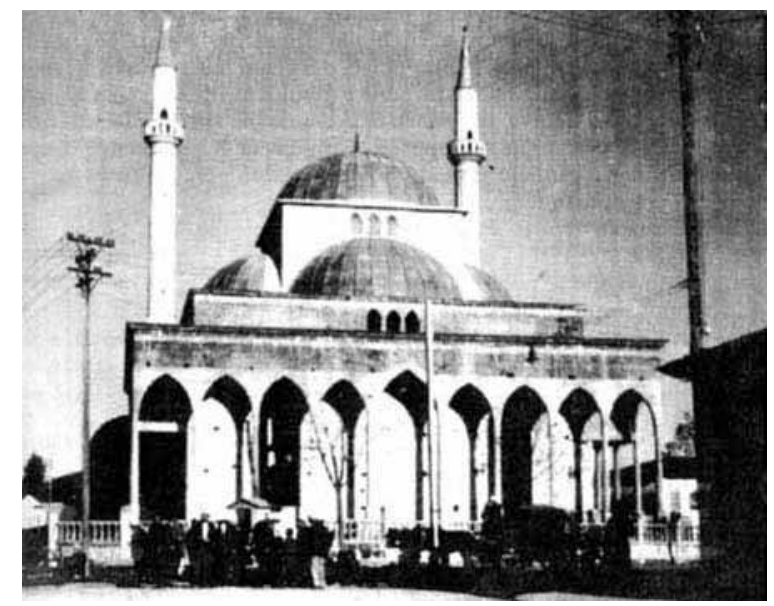

Mosquée de Parruca (Shkodra), dont la construction fut achevée en 1943-1944 (Source : Bushati, $1998: 125$ )

\section{Conclusion}

Malgré une politique visant à limiter la présence du religieux dans l'espace publique (en particulier à l'école), politique qui rejoint partiellement l'idée des réformateurs musulmans de rationaliser le réseau des mosquées en supprimant ce qu'ils appellent " les mosquées inutiles », une politique active - bien que limitée - de construction de mosquées ou de soutien à la construction de mosquées est menée par les institutions islamiques albanaises ainsi que par le roi Zog, à partir de la fin des années vingt, et ceci dans un contexte de crise économique grave.

Même s'il s'agit, dans certains cas, de répondre à un simple besoin en lieux de culte dû à des facteurs démographiques ou matériels divers (urbanisation, installation de réfugiés, vétusté, confiscation), les enjeux de la construction de certains édifices de culte musulman dans l'Albanie de l'entre-deux-guerres relèvent également d'autres dimensions. Tout d'abord, la volonté de construire la légitimité de l'existence de l'État albanais en Europe à travers l'affirmation d'un islam réformé et moderne (Clayer, 2008). Les quelques grandes mosquées, en béton, devaient ainsi symboliser cet islam par leur architecture. Édifiées dans des ports, Durrës, Saranda, ou dans la capitale, elles devaient apparaître immédiatement à l'étranger qui venait dans le pays, et lui donner une autre image que celle d'un pays musulman «traditionnel » et « retardé ».

15. Voir aussi AQSh, F. 882, année 1937, dossier 1, rapport de Behxhet Shapati sur l'activité de la CI du $1^{\text {er }}$ mai 1937. 
Mais l'Autre n'était pas que l'étranger débarqué sur les côtes ou dans la capitale albanaise ; il était aussi le chrétien auquel était associée l'idée d'Occident et de progrès. Les concepteurs des projets de nouvelles mosquées, institutionnels ou non, voulaient également rehausser le prestige de l'islam aux yeux de la population chrétienne, non sans un certain esprit de compétition. D’autant que les réseaux des églises, catholiques et orthodoxes, étaient en partie plus récents que celui des mosquées. De façon très particulière, à la frontière grecque, l'enjeu devenait national. L'affirmation d'une religion moderne devenait en effet une affirmation nationale vis-à-vis de la Grèce et de ses revendications territoriales, réelles ou imaginées, qui s'appuyaient sur une présence chrétienne orthodoxe dans le Sud de l'Albanie.

Au plan national, il est intéressant de noter que l'édification de mosquées a également servi à construire le prestige du roi Zog (intronisé en 1928, après avoir été président de la République) et de sa famille auprès des musulmans du pays ainsi que vis-à-vis de l'extérieur. Outre les mosquées de Durrës et de Saranda, associées à la famille régnante, une autre mosquée fut élevée à Burrel, dans la région d'origine de la famille Zog (ou Zogolli), en souvenir de la reinemère ${ }^{16}$. L'image du souverain " occidental ", qui mène une politique laïcisante et qui, en 1938, va même jusqu'à épouser une femme issue de la noblesse catholique hongroise, est donc à nuancer.

Enfin, il convient de relever que plusieurs de ces nouveaux lieux de culte sont prévus ou conçus comme devant être de taille importante. Il ne s'agit plus de lieux devant accueillir la djemaat, la communauté des fidèles du quartier. Ces mosquées sont désormais destinées à regrouper les musulmans d'une ville, en des occasions religieuses et/ou nationales. Les édifices doivent donc participer à former la communauté au niveau supra-local. En cela, leur réalisation sert également à bâtir la légitimité des nouvelles institutions islamiques nationales, porteuses d'un islam « réformé » et "moderne ». Si l'on y regarde de plus près, on s'aperçoit cependant que la faiblesse financière et structurelle de la communauté islamique l'empêche d'être l'actrice principale de cet effort de construction. La famille royale, les institutions islamiques régionales, voire les simples fidèles sont autant de moteurs qui poussent à, ou qui permettent l'édification de nouveaux lieux de culte. Preuve que les enjeux et les acteurs de la construction de lieux de culte sont multiples et que le champ religieux évolue selon des dynamiques et des rapports de force complexes.

\author{
Nathalie CLAYER \\ CNRS-EHESS, Paris \\ clayer@ehess.fr
}

16. La mosquée fut inaugurée en présence du roi Zog, le 23 juillet 1937 (Zani i naltë, XII/8, p. 225). 


\section{Bibliographie}

Bushati Hamdi, 1998, Shkodra dhe motet [Shkodra à travers le temps], t. 1, Shkodër, Rozafat.

Clayer Nathalie, 2007, Aux origines du nationalisme albanais, Paris, Karthala.

-, 2008, "Behind the Veil. The reform of Islam in inter-war Albania or the search for a "modern" and "European" Islam ", in Clayer N., Germain E., Islam in Inter-War Europe, London, Hurst, pp. 128-155.

-, 2009a, "L'albanisation des toponymes dans l'Albanie de l'entre-deux-guerres ou les méandres d'une lente construction étatique ", in de Rapper G., Sintès P., Nommer et classer dans les Balkans, Athènes, École française d'Athènes, pp. 237-255.

-, 2009b, "The Tijaniyya. Islamic reformism and Islamic revival in inter-war Albania ", Journal of Muslim Minority Affairs, 29-4, pp. 483-493.

FisCHER Bernd Jürgen, 1984, King Zog and the Struggle for Stability in Albania, Boulder, East European Monographs.

HaSANBEgović Zlatko, 2007, Muslimani u Zagrebu 1878-1945: Doba utemeljenja, Zagreb, Institut društvenih znanosti Ivo Pilar.

MAYeur Jean-Marie, 1997, La question lä̈que, XIX ${ }^{e}-X^{e}$ siècle, Paris, Fayard.

Morrozo della Rocca Roberto, 1990, Nazione e Religione in Albania (1920-1944), Bologna, Il Mulino.

NeCiPOĞLu Gülrü, 1982, «The Life of an Imperial Monument: Hagia Sophia after Byzantium ", in Mark R., Cakmak A., Hagia Sophia from the Age of Justinian to the Present, Cambridge, Cambridge University Press, pp. 195-225.

SAn Nicolo Mariano, 1918, Die Verwaltung Albaniens durch die k.u.k. österreichischungarischen Truppen in den ersten zwei Jahren der Besetzung des Landes an der Hand der ergangenen Befehle, Wien, Kriegsministerium.

SHARofr Haki, 1931, "Rinija kërkon Reforma Dogmatike? Feja Islame s'âsht Dogmatike » [La jeunesse demande des réformes dogmatiques ? La religion islamique n'est pas dogmatique], Zani i naltë [La voix suprême], VI/1, pp. 32-36.

SJÖBERG Örjan, 1991, Rural Change and Development in Albania, Boulder, Westview Press. VuÇITERNi Salih, 1927, "Deklaratë mbi nevojën e reformave » [Déclaration sur le besoin de réformes], Zani i naltë, IV/7, pp. 193-207.

YavuZ Hakan, 2003, Islamic Political Identity in Turkey, Oxford, Oxford University Press.

\section{Résumé}

Plusieurs mosquées relativement importantes ont été construites dans l'Albanie de l'entre-deux-guerres. À travers différents cas, cet article analyse les enjeux de ces constructions réalisées dans un contexte de crise économique et de volonté de "rationaliser " le réseau des lieux de culte islamiques dans le pays : volonté de construire la légitimité de l'existence de l'État albanais en Europe à travers l'affirmation d'un islam réformé et moderne; rehausser le prestige de l'islam aux yeux de la population chrétienne, non sans un certain esprit de compétition; affirmer la souveraineté albanaise à la frontière grecque; construire le prestige du roi Zog et de sa famille auprès des musulmans $d u$ pays; construire une communauté musulmane au niveau supralocal et bâtir la légitimité des nouvelles institutions islamiques nationales.

Mots-clés : Albanie, Europe, islam, mosquées, nation, laïcité. 


\section{Abstract}

Several relatively large mosques were built in Albania between the two world wars. Through various cases, this article analyzes the challenges of these constructions made in a context of economic crisis and desire to "rationalize" the network of Islamic places of worship in the country: desire to build the legitimacy of the existence of the Albanian state in Europe through the affirmation of an reformed and modern Islam; wish to enhance the prestige of Islam in the eyes of the Christian population, not without a certain competitive spirit; necessity to affirm the sovereignty of Albania to the Greek border; attempt to build the prestige of King Zog and his family among the Muslims of the country; wish to build a Muslim community at the supra-local level and to enhance the legitimacy of the new national Islamic institutions.

Key words: Albania, Europe, Islam, mosques, nation, secularism.

\section{Resumen}

Numerosas mezquitas relativamente importantes han sido construidas en la Albania de entre guerras. A través de diferentes casos, este artículo analiza lo que está en juego en estas construcciones hechas en un contexto de crisis económica y de la voluntad de "racionalizar" la red de los lugares de culto islámicos en el país: voluntad de construir la legitimidad de la existencia del Estado albanés en Europa a través de la afirmación de un Islam reformado y moderno, realzar el prestigio del Islam a los ojos de la población cristiana, no sin un cierto espíritu de competencia, afirmar la soberanía albanesa en la frontera griega, construir el prestigio del Rey Zog y de su familia ante los musulmanes del país, construir una comunidad musulmana a nivel supra-local y cimentar la legitimidad de las nuevas instituciones islámicas nacionales.

Palabras clave: Albania, Europa, Islam, mezquitas, laicidad. 